\title{
Quantum coherence of the molecular states and their corresponding currents in nanoscale Aharonov-Bohm interferometers
}

\author{
Jian-Heng Liu, ${ }^{1}$ Matisse Wei-Yuan Tu, ${ }^{1,2}$ and Wei-Min Zhang ${ }^{1, *}$ \\ ${ }^{1}$ Department of Physics and Centre for Quantum Information Science, National Cheng Kung University, Tainan 70101, Taiwan \\ ${ }^{2}$ Department of Physics and Center of Theoretical and Computational Physics, University of Hong Kong, Hong Kong \\ (Received 9 January 2016; revised manuscript received 22 June 2016; published 5 July 2016)
}

\begin{abstract}
By considering a nanoscale Aharonov-Bohm (AB) interferometer consisting of a laterally coupled double dot coupled to the source and drain electrodes, we investigate the $\mathrm{AB}$ phase dependence of the bonding and antibonding states and the transport currents via the bonding and antibonding state channels. The relations of the $\mathrm{AB}$ phase dependence between the quantum states and the associated transport current components are analyzed, which provides useful information for the reconstruction of quantum states through the measurement of the transport current in such systems. We also obtain the validity of the experimental analysis [given in T. Hatano et al., Phys. Rev. Lett. 106, 076801 (2011)] that bonding state currents in different energy configurations are almost the same. With the coherent properties in the quantum dot states as well as in the transport currents, we also provide a way to manipulate the bonding and antibonding states through the $\mathrm{AB}$ magnetic flux.
\end{abstract}

DOI: 10.1103/PhysRevB.94.045403

\section{INTRODUCTION}

Quantum coherence of electrons in nanostructures is expected to manage quantum computation and quantum information. It is essential to prepare and read out the state of the qubit in quantum information processing. There have been many experiments and theoretical analyses on quantum coherence manipulation of electron states in double quantum dots (DQDs) which are thought to be a promising charge qubit [1-14]. The techniques to reconstruct quantum states from series of measurements about the system are known as quantum state tomography [15-17]. Quantum state tomography is resource demanding and it aims at very detailed description of coherence of quantum states, see, for example $[18,19]$. On the other hand, transport measurement utilizing quantum interference has revealed the main coherent properties of traveling electrons. How the latter can be associated with the coherence of local quantum dot states of the DQDs is worthy of further investigation.

Quantum coherence has been detected through the Aharonov-Bohm (AB) interference [20]. Double quantum dots embedded in $\mathrm{AB}$ geometry were achieved in Refs. [21-23]. The AB phase coherence of electrons through each dot would induce oscillating current as a function of the magnetic flux, which is simply called the AB oscillation in the literature. The results show that the $\mathrm{AB}$ phase coherence can be easily manipulated in these devices. In Coulomb blockade and cotunneling regimes, it is predicted theoretically that currents through spin-singlet and triplet states carry $\mathrm{AB}$ phases with a half of period difference [24]. For one-electron states, the half-period difference of $\mathrm{AB}$ oscillation is also anticipated in transport currents through the bonding and antibonding state channels $[25,26]$, demonstrated in electron conductance. In particular, the authors of Ref. [25] revealed that there are two resonances, the Breit-Wigner resonance and the Fano resonance, in the electron conductance that are associated to the bonding

\footnotetext{
*wzhang@mail.ncku.edu.tw
}

and antibonding states and the interference between them. Furthermore, the authors of Ref. [26] investigated the electron transport in such system by varying the indirect coupling between double dots via the leads at zero temperature, and they found that the Fano resonance can be suppressed as the indirect coupling strength decreases, and the remaining Breit-Wigner resonance contains two peaks associated with the bonding and the antibonding states, respectively. Motivated with these theoretical investigations, the transport currents passing through the bonding and antibonding state channels have been detected experimentally [27], where the half-period difference of $\mathrm{AB}$ oscillation in electron currents through the bonding and antibonding state channels, respectively, is thought to be resulted from the parity of the wave functions of the bonding and antibonding states, which is a property of the device geometry.

Inspired by the ability of detecting currents from different channels, we attempt to investigate the coherent properties of quantum states in DQDs, for which the corresponding currents must have a direct connection [25,26]. In Ref. [27], two different energy configurations are used, which are succeeded by two different gate voltage settings. The transport currents under these two configurations are measured. The measured currents are used to determine the bonding and/or antibonding state channels in one of the configurations. In Ref. [27], the authors assumed that the transport currents flowing through the bonding state channel in different energy configuration are almost the same. We would first verify the validity of this assumption, using our theoretical framework of the quantum transport theory based on the master-equation approach [28-31]. We then investigate the AB oscillation dependence between the bonding and antibonding states and the transport currents flowing through the corresponding channels, from which we further explore the quantum coherence between the two quantum dot states. The results provide the full information for the reconstruction of quantum states of the promising charge qubit in terms of two physical dot states through the measurements of transport current. Finally, we discuss the way to manipulate the bonding and antibonding states of DQDs with the AB magnetic flux. 
The rest of the paper is organized as follow: In Sec. II, we begin with the system of DQDs coupled to two leads to study the reduced density matrix of the DQD system and the transport currents flowing through the bonding and antibonding state channels. In Sec. III, we obtain the condition for the validity of the method used in Ref. [27] in analyzing the connection of the bonding and antibonding states with the measured currents. In Sec. IV, we discuss the correspondence of the AB phase dependence between the reduced density matrix of the bonding and antibonding states with the associated transport currents, and the relation to the quantum coherence between the two physical dot bases as the promising charge qubit. A way to manipulate the bonding and antibonding states with the $\mathrm{AB}$ magnetic flux is also given. Finally, a summary is presented in Sec. V.

\section{COUPLED DQDS MOLECULE}

We consider a nanosystem of two laterally coupled singlelevel QDs coupled to two leads. The Hamiltonian of the system is given by

$$
H=H_{\mathrm{DQD}}+H_{B}+H_{T},
$$

where $H_{\mathrm{DQD}}$ is the Hamiltonian of the DQDs

$$
H_{\mathrm{DQD}}=\sum_{i=1}^{2} \epsilon_{i j} d_{i}^{\dagger} d_{j},
$$

and $d_{i}\left(d_{i}^{\dagger}\right)$ is the annihilation (creation) operator in the $i$ th QD, $\epsilon_{i i}$ is the energy level of the $i$ th $\mathrm{QD}$, and $\epsilon_{i j}$ with $i \neq j$ is the tunneling matrix element between the DQDs. The Hamiltonian of the two leads is given by $H_{B}$ :

$$
H_{B}=\sum_{\alpha=L, R} \sum_{k} \varepsilon_{\alpha k} c_{\alpha k}^{\dagger} c_{\alpha k},
$$

where the label $\alpha$ denotes the left or the right lead, and $c_{\alpha k}\left(c_{\alpha k}^{\dagger}\right)$ is the annihilation (creation) operator of the $k$ th level in lead $\alpha$. The Hamiltonian $H_{T}$ describes the tunnelings between the QDs and the leads:

$$
H_{T}=\sum_{\alpha=L, R} \sum_{i=1}^{2} \sum_{k}\left(V_{i \alpha k} d_{i}^{\dagger} c_{\alpha k}+\text { H.c. }\right) .
$$

By threading a magnetic flux $\Phi$ to the above system, the tunneling matrix elements would carry an $\mathrm{AB}$ phase, $V_{i \alpha k}=$ $\bar{V}_{i \alpha k} e^{i \phi_{i \alpha}}, \phi_{i \alpha}$ is the $\mathrm{AB}$ phase when the electron tunnels from lead $\alpha$ to the $i$ th dot, and $\bar{V}_{i \alpha k}$ is a real tunneling amplitude. The $\mathrm{AB}$ phase will also affect on $H_{\mathrm{DQD}}$, i.e., for $i \neq j, \epsilon_{i j}=\bar{\epsilon}_{i j} e^{i \phi_{i j}}$ where $\bar{\epsilon}_{i j}=-t_{c}$ is a real amplitude and $\phi_{i j}$ is the AB phase from $j$ th dot to $i$ th dot. The relation of the $\mathrm{AB}$ phases with the magnetic flux $\Phi$ is given by $\phi_{1 L}-\phi_{1 R}+\phi_{2 R}-\phi_{2 L}=$ $2 \pi \Phi / \Phi_{0}=\varphi$, where $\Phi_{0}$ is the flux quanta. We also set $\phi_{12}=0$ according to Refs. [25-27].

In order to study the molecular states of the DQDs, we may change the basis by diagonalizing $H_{\mathrm{DQD}}$. By labeling the bonding state and the antibonding state with the signs - and + respectively, the Hamiltonian of the DQDs becomes

$$
H_{\mathrm{DQD}}=\sum_{\nu= \pm} \epsilon_{\nu} d_{\nu}^{\dagger} d_{\nu}
$$

where $\epsilon_{ \pm}$are the corresponding energy levels, and $d_{ \pm}\left(d_{ \pm}^{\dagger}\right)$ are the associated annihilation (creation) operators, which are given by

$$
\begin{aligned}
\epsilon_{ \pm} & =\frac{1}{2}\left[\left(\epsilon_{11}+\epsilon_{22}\right) \pm \sqrt{\left(\epsilon_{11}-\epsilon_{22}\right)^{2}+4 t_{c}^{2}}\right], \\
\left(\begin{array}{l}
d_{+} \\
d_{-}
\end{array}\right) & =\left(\begin{array}{cc}
\cos \frac{\theta}{2} & -\sin \frac{\theta}{2} \\
\sin \frac{\theta}{2} & \cos \frac{\theta}{2}
\end{array}\right)\left(\begin{array}{l}
d_{1} \\
d_{2}
\end{array}\right),
\end{aligned}
$$

and $\tan \theta=2 t_{c} /\left(\epsilon_{11}-\epsilon_{22}\right)$.

\section{A. Density matrix of the DQDs}

The reduced density matrix $\rho(t)=\operatorname{Tr}_{E}\left[\rho_{\text {tot }}(t)\right]$ that describes quantum states of the DQDs can be solved from the exact master equation we derived previously by tracing over all the reservoir states [28-31]. The explicit form of the exact master equation for the given total Hamiltonian (1) is presented in the Appendix. By denoting the empty state with $|0\rangle$, the bonding and antibonding states with $|\nu\rangle:=| \pm\rangle$, and the doubly occupied state by $|d\rangle$, the reduced density matrix elements at the later time $t$ for an arbitrary initial DQD state that are solved explicitly from the master equation (A1) in the Appendix are given as follows [32]:

$$
\begin{aligned}
\rho_{00}(t)= & A(t)\left\{\rho_{00}\left(t_{0}\right)+\rho_{d d}\left(t_{0}\right) \operatorname{det}\left[J_{3}(t)\right]\right. \\
& \left.-\sum_{v, v^{\prime}=: \pm} \rho_{v v^{\prime}}\left(t_{0}\right) J_{3 v^{\prime} v}(t)\right\}, \\
\rho_{++}(t)= & 1-\rho_{00}(t)-\rho_{--}^{(1)}(t), \quad \rho_{+-}(t)=\rho_{+-}^{(1)}(t), \\
\rho_{--}(t)= & 1-\rho_{00}(t)-\rho_{++}^{(1)}(t), \quad \rho_{-+}(t)=\rho_{+-}^{*}(t), \\
\rho_{d d}(t)= & 1-\rho_{00}(t)-\rho_{++}(t)-\rho_{--}(t),
\end{aligned}
$$

and the other off-diagonal density matrix elements between the different states are all zero, where $A(t)=\operatorname{det}[\boldsymbol{I}-\boldsymbol{v}(t, t)]$, $\boldsymbol{J}_{3}(t)=\boldsymbol{u}^{\dagger}\left(t, t_{0}\right)[\boldsymbol{I}-\boldsymbol{v}(t, t)]^{-1} \boldsymbol{u}\left(t, t_{0}\right)-\boldsymbol{I}$, and $\boldsymbol{I}$ is the identity matrix, $\rho\left(t_{0}\right)$ is the initial reduced density matrix, the singleparticle reduced density matrix $\rho^{(1)}(t)$ of the DQDs is [29]

$$
\begin{aligned}
\rho_{\nu \nu^{\prime}}^{(1)}(t) & =\operatorname{Tr}_{S}\left[a_{\nu^{\prime}}^{\dagger} a_{\nu} \rho(t)\right] \\
& =\left[\boldsymbol{u}\left(t, t_{0}\right) \rho^{(1)}\left(t_{0}\right) \boldsymbol{u}^{\dagger}\left(t, t_{0}\right)+\boldsymbol{v}(t, t)\right]_{v v^{\prime}},
\end{aligned}
$$

and $\boldsymbol{u}\left(t, t_{0}\right)$ and $\boldsymbol{v}(t, t)$ are the nonequilibrium Green functions that fully characterize the electron dissipation and fluctuations in the master equation and can be solved from Eqs. (A3) and (A4).

The solution (7) of the reduced density matrix for the DQDs can be further specified under the experimental conditions given in Ref. [27], namely, the energy of each dot $\epsilon_{11}=\epsilon_{22}=$ $\epsilon_{0}$, the spectral density of Eq. (A6) for lead $\alpha, \boldsymbol{\Gamma}_{\alpha}(\varepsilon)=\boldsymbol{\Gamma}_{\alpha}$ (wide band limit) with the level broadenings of the left lead $\Gamma_{L 11}=\Gamma_{L 22}=\Gamma_{L}$ and the right lead $\Gamma_{R 11}=\Gamma_{R 22}=\Gamma_{R}$, plus the indirect interdot couplings of the left lead $\Gamma_{L 12}=$ $a_{L} \Gamma_{L} e^{i \frac{\varphi}{2}}$ and the right lead $\Gamma_{R 12}=a_{R} \Gamma_{R} e^{-i \frac{\varphi}{2}}$, where the indirect coupling parameter $a_{L, R}$ was originally introduced in Ref. [26] in order to characterize the strength of the indirect coupling between two quantum dots via leads. In the molecular basis, the energies of the bonding and antibonding states are 
$\epsilon_{ \pm}=\epsilon_{0} \pm\left|t_{c}\right|$. In the above settings, the annihilation operators of the bonding and antibonding states become

$$
\left(\begin{array}{l}
d_{+} \\
d_{-}
\end{array}\right)=\frac{1}{\sqrt{2}}\left(\begin{array}{cc}
1 & -1 \\
1 & 1
\end{array}\right)\left(\begin{array}{l}
d_{1} \\
d_{2}
\end{array}\right) .
$$

The tunneling Hamiltonian between the molecular states and the leads is

$$
H_{T}=\sum_{\alpha=L, R} \sum_{ \pm} \sum_{k}\left(V_{ \pm \alpha k} d_{ \pm}^{\dagger} c_{\alpha k}\right),
$$

with the tunneling matrix elements

$$
\left(\begin{array}{l}
V_{+\alpha k} \\
V_{-\alpha k}
\end{array}\right)=\frac{1}{\sqrt{2}}\left(\begin{array}{cc}
1 & -1 \\
1 & 1
\end{array}\right)\left(\begin{array}{l}
V_{1 \alpha k} \\
V_{2 \alpha k}
\end{array}\right) .
$$

The level broadening matrix $\boldsymbol{\Gamma}_{\alpha}$ is then given by

$$
\boldsymbol{\Gamma}_{L, R}=\left(\begin{array}{ll}
\Gamma_{++} & \Gamma_{+-} \\
\Gamma_{-+} & \Gamma_{--}
\end{array}\right)_{L, R}=\Gamma_{L, R}\left(\boldsymbol{I}-\vec{\alpha}_{L, R} \cdot \overrightarrow{\boldsymbol{\sigma}}\right),
$$

where $\quad \vec{\alpha}_{L, R}=\left(\alpha_{L, R}^{x}, \alpha_{L, R}^{y}, \alpha_{L, R}^{z}\right)=a_{L, R}\left(0, \mp \sin \frac{\varphi}{2}, \cos \frac{\varphi}{2}\right)$ and $\vec{\sigma}$ are the Pauli matrices.

Then, the Green function $\boldsymbol{u}\left(t, t_{0}\right)$ has a simple solution:

$$
\begin{aligned}
\boldsymbol{u}\left(t, t_{0}\right) & =\left(\begin{array}{ll}
u_{++}\left(t, t_{0}\right) & u_{+-}\left(t, t_{0}\right) \\
u_{-+}\left(t, t_{0}\right) & u_{--}\left(t, t_{0}\right)
\end{array}\right) \\
& =\exp \left[\left(-i \epsilon-\frac{1}{2} \boldsymbol{\Gamma}_{L}-\frac{1}{2} \boldsymbol{\Gamma}_{R}\right)\left(t-t_{0}\right)\right],
\end{aligned}
$$

where $\epsilon=\left(\begin{array}{cc}\epsilon_{+} & 0 \\ 0 & \epsilon_{-}\end{array}\right)$. In the energy domain, it is given by

$$
\mathbf{G}^{r}(\varepsilon)=-i \int_{0}^{\infty} e^{i \varepsilon t} \boldsymbol{u}(t) d t=\left(\boldsymbol{\epsilon}-\varepsilon \boldsymbol{I}+\frac{i}{2} \boldsymbol{\Gamma}\right)^{-1},
$$

with $\boldsymbol{\Gamma}=\boldsymbol{\Gamma}_{L}+\boldsymbol{\Gamma}_{R}$. The Green function $\boldsymbol{v}$ in the steady-state limit is

$$
\boldsymbol{v}=\int_{-\infty}^{\infty} \frac{d \varepsilon}{2 \pi} \sum_{\alpha} f_{\alpha}(\varepsilon) \mathbf{G}^{r}(\varepsilon) \boldsymbol{\Gamma}_{\alpha} \mathbf{G}^{a}(\varepsilon),
$$

and $\mathbf{G}^{a}(\varepsilon)=\left[\mathbf{G}^{r}(\varepsilon)\right]^{\dagger}$.

As one can see, in the steady-state limit, $\boldsymbol{u}(t \rightarrow \infty)=0$ so that the single-particle reduced density matrix of the DQDs is reduced to

$$
\rho_{\nu v^{\prime}}^{(1)}(t \rightarrow \infty)=[\boldsymbol{v}]_{v v^{\prime}},
$$

where $\boldsymbol{v}$ is given by Eq. (15). Following the experiment [27], the initial DQDs are empty so that $\rho_{00}\left(t_{0}\right)=1$ and other initial density matrix elements of the DQDs all equal to zero. Then, Eq. (7) in the steady-state limit can be simplified to

$$
\begin{aligned}
\rho_{00} & =\operatorname{det}[\boldsymbol{I}-\boldsymbol{v}], \\
\rho_{++} & =1-\rho_{00}-\boldsymbol{v}_{--}, \quad \rho_{+-}=\boldsymbol{v}_{+-}, \\
\rho_{--} & =1-\rho_{00}-\boldsymbol{v}_{++}, \quad \rho_{-+}=\boldsymbol{v}_{+-}^{*}, \\
\rho_{d d} & =\operatorname{det}[\boldsymbol{v}] .
\end{aligned}
$$

Thus, the reduced density matrix elements of the DQDs are fully determined by the Green function solution (15) through the solution (14).

\section{B. Quantum transport current}

The quantum transport current of electrons flowing from lead $\alpha$ into the DQDs is defined by

$$
I_{\alpha}(t)=-e \frac{d}{d t} \operatorname{Tr}_{S \otimes R}\left[\rho_{\mathrm{tot}}(t) N_{\alpha}\right],
$$

where $N_{\alpha} \equiv \sum_{k} c_{\alpha k}^{\dagger} c_{\alpha k}$. Using the master equation given in the Appendix, the transport current can also be expressed in terms of the Green functions $\boldsymbol{u}\left(t, t_{0}\right)$ and $\boldsymbol{v}(\tau, t)$ as well [28-31]:

$$
\begin{aligned}
I_{\alpha}(t)= & -2 e \operatorname{Re} \operatorname{Tr} \int_{t_{0}}^{t} d \tau\left\{\boldsymbol{g}_{\alpha}(t, \tau) \boldsymbol{u}\left(\tau, t_{0}\right) \rho^{(1)}\left(t_{0}\right) \boldsymbol{u}^{\dagger}\left(t, t_{0}\right)\right. \\
& \left.+\boldsymbol{g}_{\alpha}(t, \tau) \boldsymbol{v}(\tau, t)-\tilde{\boldsymbol{g}}_{\alpha}(t, \tau) \boldsymbol{u}^{\dagger}(t, \tau)\right\} .
\end{aligned}
$$

This expression of the transport currents can also be derived directly from Keldysh's Green function technique [33], but the dependence of initial conditions, i.e., the first term in Eq. (19), was omitted in Ref. [33]. The net transport current measured in experiments is given by

$$
I(t)=\frac{1}{2}\left[I_{L}(t)-I_{R}(t)\right]
$$

The steady-state electron current in the wide band limit can be reduced to

$$
I_{\alpha}=-2 e \operatorname{Re} \operatorname{Tr}\left\{\frac{1}{2} \boldsymbol{\Gamma}_{\alpha} \boldsymbol{v}-i \int_{-\infty}^{\infty} \frac{d \varepsilon}{2 \pi} f_{\alpha}(\varepsilon) \boldsymbol{\Gamma}_{\alpha} \mathbf{G}^{r}(\varepsilon)\right\} .
$$

Carrying out explicitly the real part of Eq. (21), the transport current of Eq. (20) in the steady-state limit obeys the generalized Landauer-Büttiker formula

$$
I=\frac{e}{2 \pi} \int d \varepsilon\left[f_{L}(\varepsilon)-f_{R}(\varepsilon)\right] T(\varepsilon),
$$

where the electron transmission is

$$
T(\varepsilon)=\operatorname{Tr}\left[\mathbf{G}^{a}(\varepsilon) \boldsymbol{\Gamma}_{R} \mathbf{G}^{r}(\varepsilon) \boldsymbol{\Gamma}_{L}\right] .
$$

According to the analyses in Ref. [27], the total transport current can be divided into components flowing through the bonding and antibonding state channels, plus the interference between them:

$$
I=I_{+}+I_{-}+I_{+-} .
$$

These current components are explicitly given by

$$
\begin{aligned}
I_{ \pm}= & \frac{e}{2 \pi} \int_{-\infty}^{+\infty} d \varepsilon\left[f_{L}(\varepsilon)-f_{R}(\varepsilon)\right] \Gamma_{L \pm \pm} \Gamma_{R \pm \pm}\left|G_{ \pm \pm}^{r}(\varepsilon)\right|^{2}, \\
I_{+-}= & \frac{e}{2 \pi} \int_{-\infty}^{+\infty} d \varepsilon\left[f_{L}(\varepsilon)-f_{R}(\varepsilon)\right]\left\{\Gamma_{L++} \Gamma_{R--}\left|G_{-+}^{r}\right|^{2}\right. \\
& +\Gamma_{L--} \Gamma_{R++}\left|G_{+-}^{r}\right|^{2}+2 \operatorname{Re}\left\{G_{++}^{a} \Gamma_{R++} G_{+-}^{r} \Gamma_{L-+}\right. \\
& +G_{+-}^{a} \Gamma_{R-+} G_{++}^{r} \Gamma_{L++}+G_{+-}^{a} \Gamma_{R-+} G_{+-}^{r} \Gamma_{L-+} \\
& +G_{++}^{a} \Gamma_{R+-} G_{--}^{r} \Gamma_{L-+}+G_{+-}^{a} \Gamma_{R--} G_{--}^{r} \Gamma_{L-+} \\
& \left.\left.+G_{--}^{a} \Gamma_{R-+} G_{+-}^{r} \Gamma_{L--}\right\}\right\},
\end{aligned}
$$

where $\Gamma_{L \pm \pm} \Gamma_{R \pm \pm}\left|G_{ \pm \pm}^{r}(\varepsilon)\right|^{2}$ are the effective transmission coefficients of the bonding (antibonding) state channels. The transport current component $I_{+-}$is the second-order term of $a_{L, R}$, and hence its contribution to the total transport current is ignorable in the weak indirect coupling limit [27] $I_{+-} \simeq 0$. 


\section{TRANSPORT CURRENT THROUGH THE BONDING AND ANTIBONDING STATE CHANNELS}

In Refs. [25,26], transport properties of the same system, the $\mathrm{AB}$ interferometer embedded with DQDs, was analyzed through the resonance tunneling. The authors of Ref. [25] found two resonances in the electron conductance near the bonding and antibonding states that are composed of a BreitWigner resonance and a Fano resonance, for which the widths of the Breit-Wigner resonance and the Fano factor are sensitive to the AB phase. The authors of Ref. [26] explored further the associated resonances in the conductance with the molecular states by investigating the indirect coupling effect on the resonances. They found that the full destructive interference of the Fano resonance only happens for the strongest indirect coupling $\left|a_{L, R}\right|=1$. When $\left|a_{L, R}\right|$ decreases from 1 to 0 , the Fano resonance is gradually suppressed, and the remaining result is the Breit-Wigner resonance containing two peaks associated with the bonding and antibonding states. In our formalism, $I_{ \pm}$are the transport currents flowing through the bonding and antibonding state channels, respectively, which gives the two peaks in the electron conductance for Breit-Wigner resonance, as shown in Ref. [26], and $I_{+-}$ is the transport current due to interference between the bonding and antibonding state channels, which induce Fano resonance in the electron conductance when $\left|a_{L, R}\right| \rightarrow 1$, as shown in Refs. [25,26]. The transport currents flowing through the bonding and antibonding state channels were explicitly detected later [27]. The theoretical analysis in Ref. [26] and the experimental analysis in Ref. [27] inspire us to find the explicit relation between the DQD reduced density matrix elements and the transport currents in the molecular state basis.

In the experiment [27], the electron currents are measured under two different energy configurations for the bonding and antibonding state channels with the fixed bias and indirect interdot weak couplings, as shown in Fig. 1(a). Other parameter settings in Ref. [27] are as follows: the level broadenings of the left lead $\Gamma_{L}=0.3 \Gamma$ and the right lead $\Gamma_{R}=0.7 \Gamma\left(\Gamma=\Gamma_{L}+\Gamma_{R}\right)$, the indirect interdot coupling parameters $a_{L}=-0.1$ for the left lead and $a_{R}=0.15$ for the right lead, the direct interdot coupling $t_{c}=-60 \Gamma$, the chemical potentials of the left lead $\mu_{L}=125 \Gamma$ and the right lead $\mu_{R}=-125 \Gamma$, and the temperature of the reservoirs is set at $k_{B} T=10 \Gamma$. The measured currents are the total electron currents in each configuration. As shown by Fig. 1(a), in configuration 1 , only the energy of the bonding state locates within the bias window $\left(\mu_{L}-\mu_{R}\right)$. In configuration 2 , both the energies of the bonding and antibonding states lie in the bias window. These two energy configurations can be succeeded by tuning gate voltages.

In configuration 1 , the current flowing through the bonding state channel, denoted by $I_{1-}$, is dominant such that the total current is almost given by $I \simeq I_{1-}$, where the current $I_{1+}$ flowing through the antibonding state channel in configuration 1 is negligible. In configuration 2 , the total current $I_{2}=I_{2+}+$ $I_{2-}+I_{2+-}$, where $I_{2+}, I_{2-}$ are the currents flowing through the antibonding and bonding state channels in configuration 2 , respectively, and $I_{2+-}$ is the current due to the interference between the bonding and antibonding state channels. The latter is negligible in the weak indirect coupling regime [26]. (a)

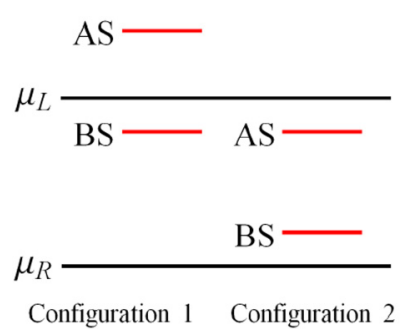

(c)

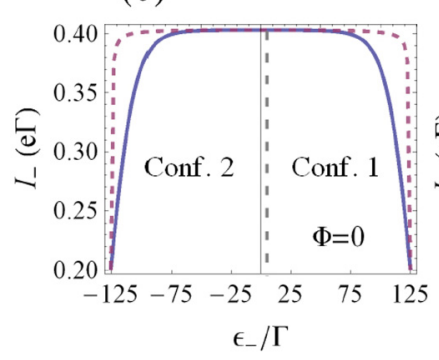

(b) $\cdots \cdots f_{L}(\varepsilon)-f_{R}(\varepsilon)---\mathrm{AS}, t_{c}=10 \Gamma$

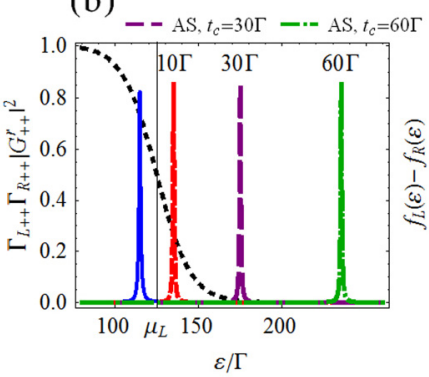

(d)

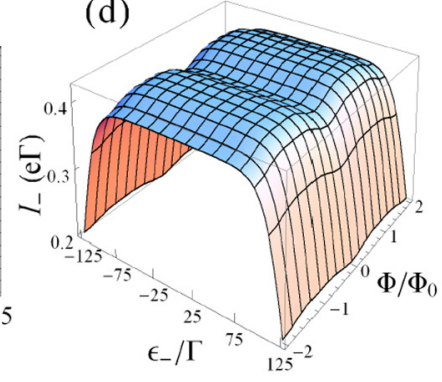

FIG. 1. (a) The schematic plot of the energy levels of the bonding and antibonding states in configurations 1 and 2 with the chemical potential of the left and right leads $\mu_{L}$ and $\mu_{R}$. (b) The difference of the left and right lead particle distributions $f_{L}(\varepsilon)-f_{R}(\varepsilon)$, and the effective transmission coefficients of the bonding and antibonding state channels in configuration 1 for different interdot coupling $t_{c}$ are plotted. In this case, the energy $\epsilon_{-}$of the bonding state is fixed at $115 \Gamma$, and the corresponding transmission is plotted with the blue line. The transmissions of the antibonding state for $t_{c}=10,30,60 \Gamma$ are plotted with the red dashed line, purple long dashed line, and green dotted-dashed line, respectively. (c) $I_{-}$as a function of $\epsilon_{-}$ is plotted. The blue solid line is for temperature $k_{B} T=10 \Gamma$, and the purple dashed line is for zero temperature. The numbers 1,2 in the plot denote the corresponding energy configurations 1 and 2 for $\left|t_{c}\right|=60 \Gamma$. (d) $I_{-}$is plotted as a function of $\epsilon_{-}$and $\Phi$.

Therefore, the total current in configuration 2 is mainly given by $I_{2} \simeq I_{2+}+I_{2-}$. With the assumption that currents flowing through the bonding state channel in configurations 1 and 2 are almost the same [27], $I_{1-} \simeq I_{2_{-}}$, one can determine the currents flowing through the bonding and antibonding state channels, respectively, by the total currents measured separately in configurations 1 and 2 . This is the method used in Ref. [27] for analyzing the currents flowing through the bonding and antibonding state channels.

For the above experimental analysis, we shall check first (1), whether the current $I_{1+}$ flowing through the antibonding state channel in configuration 1 is really negligible; and (2) what are the conditions that should be satisfied such that the assumption $I_{1-} \approx I_{2-}$ is valid. According to Eq. (25a), $I_{1+}$ depends on the overlap of the difference of particle number distributions in the two leads $f_{L}(\varepsilon)-f_{R}(\varepsilon)$, with the effective transmission coefficient of antibonding state channel $\Gamma_{L++} \Gamma_{R++}\left|G_{++}^{r}(\varepsilon)\right|^{2}$. In Fig. 1(b), the difference $f_{L}(\varepsilon)-f_{R}(\varepsilon)$ is shown by the black dashed line. We theoretically fix the energy of the bonding state $\epsilon_{-}=\epsilon_{0}-\left|t_{c}\right|$ and change the interdot coupling $t_{c}$ to compare the corresponding antibonding state channel contributions to the current. In experiments, $\epsilon_{-}$can be 
BS Transmission \& $f_{L}(\varepsilon)-f_{R}(\varepsilon)$ (Dashed line)
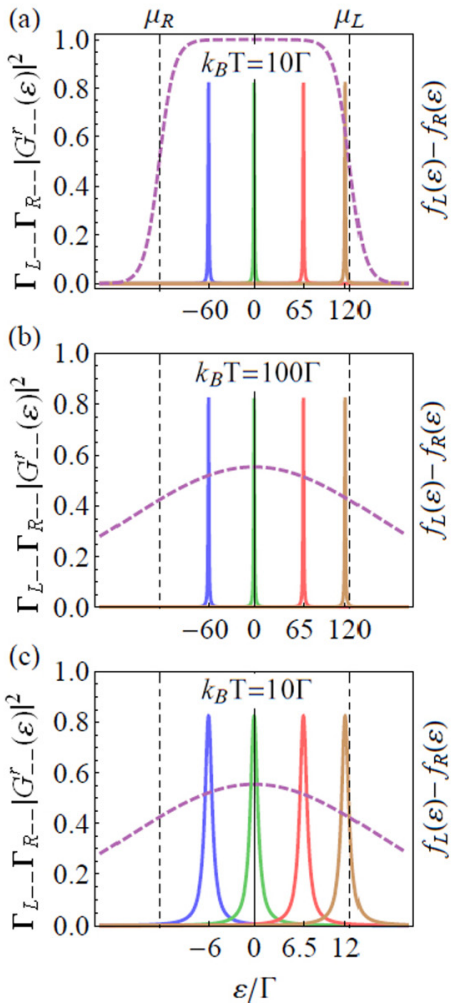

BS currents
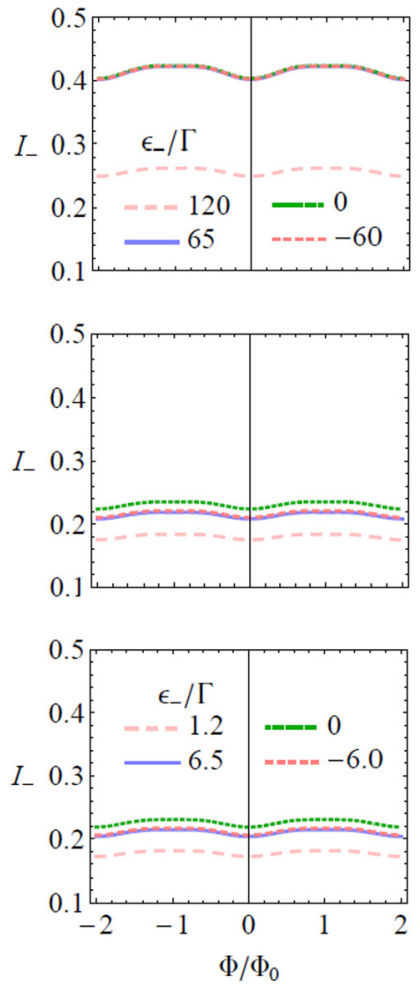

Currents

in configuration 1
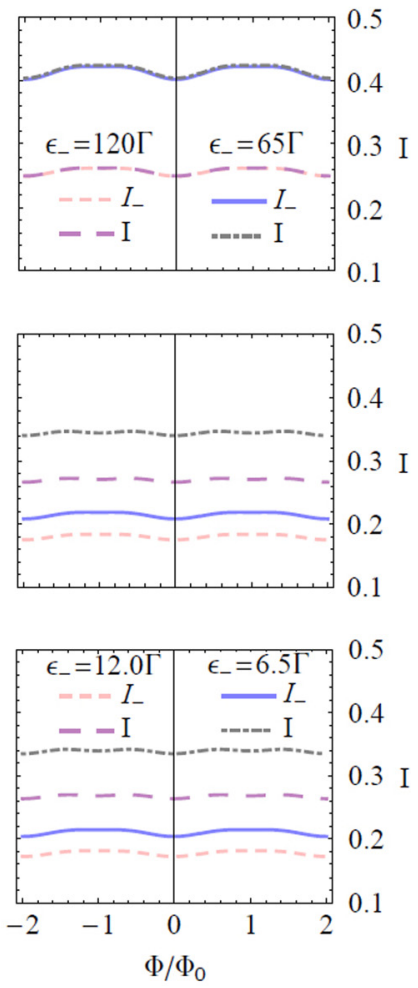

FIG. 2. The effective transmission coefficient $\Gamma_{L--} \Gamma_{R--}\left|G_{--}^{r}(\varepsilon)\right|^{2}$ of the bonding state and the difference of the left and right leads particle distribution $f_{L}(\varepsilon)-f_{R}(\varepsilon)$ for different energy of the bonding state are plotted in the left side. The $f_{L}(\varepsilon)-f_{R}(\varepsilon)$ is marked by the dashed line. The corresponding bonding currents are plotted in the middle. The bonding currents and the measured currents in configuration 1 ( $\epsilon_{-}=65,120 \Gamma$ as examples here) are plotted in the right side. (a) The original settings in Ref. [27]. (b) The high-temperature case. (c) The stronger coupling case.

manipulated through tuning the energy of DQDs and the interdot coupling simultaneously. We fix $\epsilon_{-}$so that the effective transmission coefficient $\Gamma_{L_{--}} \Gamma_{R--}\left|G_{--}^{r}(\varepsilon)\right|^{2}$ of the bonding state channel is fixed, which is shown by the blue peak in Fig. 1(b). Other peaks are the corresponding effective transmission coefficient $\Gamma_{L++} \Gamma_{R++}\left|G_{++}^{r}(\varepsilon)\right|^{2}$ of the antibonding state channel for different $t_{c}$. As shown by Fig. 1(b), the larger $t_{c}$ gives the smaller overlap of $f_{L}(\varepsilon)-f_{R}(\varepsilon)$ with $\Gamma_{L++} \Gamma_{R++}\left|G_{++}^{r}(\varepsilon)\right|^{2}$ and hence the smaller current $I_{1+}$ flowing through the antibonding state channel in configuration 1. So, we conclude that $I_{1+}$ is negligible when $t_{c}$ is properly large enough to make $\Gamma_{L++} \Gamma_{R++}\left|G_{++}^{r}(\varepsilon)\right|^{2}$ lesser overlap with $f_{L}(\varepsilon)-f_{R}(\varepsilon)$.

On the other hand, we plot the current $I_{-}$flowing through the bonding state channel as a function of the energy $\epsilon_{-}$of the bonding state in Fig. 1(c). In our numerical calculation, the parameters are set up according to Ref. [27]. Figure 1(c) shows that the current $I_{-}$flowing through the bonding state channel becomes maximum when the energy $\epsilon_{-}$of the bonding state is located in the middle of the bias window. The current $I_{-}$ symmetrically and dramatically decays when $\epsilon_{-}$approaches closely to $\mu_{L}$ or $\mu_{R}$. Meanwhile, we fix $\left|t_{c}\right|=60 \Gamma$ here so that the energy difference of the bonding and antibonding states is fixed. Then, we can use $\epsilon_{-}$to determine which energy configuration is examined. In Fig. 1(c), the blue solid line gives the current $I_{-}$as a function of $\epsilon_{-}$for temperature $k_{B} T=$
$10 \Gamma$. It shows that $I_{-}$is almost a constant within $\left|\epsilon_{-}\right| \lesssim 80 \Gamma$. This indicates that the condition $I_{1-} \simeq I_{2-}$ is well satisfied for $\left|\epsilon_{-}\right| \lesssim 80 \Gamma$. We also plot $I_{-}$at zero temperature in Fig. 1(c) (the purple dashed line). In this case, the range for $I_{-}$being almost a constant is wider. Also, this flat pattern is maintained for arbitrary magnetic flux $\Phi$ [see Fig. 1(d)].

Now, we should check if this analysis can be applied to other settings. According to Eq. (25a), the magnitude of the bonding current $I_{-}$depends on the overlap between the quantities $f_{L}(\varepsilon)-f_{R}(\varepsilon)$ and $\Gamma_{L--} \Gamma_{R--}\left|G_{--}^{r}(\varepsilon)\right|^{2}$. In Fig. 2, we choose $\epsilon_{-}=-60,0,65,120 \Gamma$ as examples, where $\epsilon_{-}=65,120 \Gamma$ is in configuration 1 and $\epsilon_{-}=-60,0 \Gamma$ is in configuration 2 . Figure 2 gives the overlaps between $f_{L}(\varepsilon)-$ $f_{R}(\varepsilon)$ with $\Gamma_{L--} \Gamma_{R--}\left|G_{--}^{r}(\varepsilon)\right|^{2}$ for these different $\epsilon_{-}$(see the left column). The bonding currents in configurations 1 and 2 are also shown in Fig. 2 (the central column), and the bonding currents and the measured currents in configuration 1 are given in the right column in Fig. 2. Currents $I_{-}$for $\epsilon_{-}=-60,0,65,120 \Gamma$ are shown by the red small dashed line, green dotted line, blue solid line, and pink medium dashed line, respectively, and currents $I$ for $\epsilon_{-}=65,120 \Gamma$ are shown by the black dotted-dashed line and purple large dashed line, respectively. Figure 2(a) shows that the analysis works well in the original setting because $f_{L}(\varepsilon)-f_{R}(\varepsilon)=1.0$ when $\left|\epsilon_{-}\right| \lesssim 80 \Gamma$. The overlaps between $f_{L}(\varepsilon)-f_{R}(\varepsilon)$ with $\Gamma_{L--} \Gamma_{R--}\left|G_{--}^{r}(\varepsilon)\right|^{2}$ for different $\epsilon_{-}$hardly change in this 
region, and $I_{-}$and $I$ for $\epsilon_{-}=65 \Gamma$ are covered by $I_{-}$for $\epsilon_{-}=-60,0 \Gamma$.

However, when the temperature becomes higher or the couplings of DQDs to leads become stronger, as shown in Figs. 2(b) and 2(c), respectively, the overlaps between $f_{L}(\varepsilon)-$ $f_{R}(\varepsilon)$ with $\Gamma_{L--} \Gamma_{R--}\left|G_{--}^{r}(\varepsilon)\right|^{2}$ for different $\epsilon_{-}$are different. In Fig. 2(b), $f_{L}(\varepsilon)-f_{R}(\varepsilon)$ becomes broadened because of the higher temperature, and gives different overlaps with $\Gamma_{L--} \Gamma_{R--}\left|G_{--}^{r}(\varepsilon)\right|^{2}$ and hence the different contribution to $I_{-}$. On the other hand, the broadened $f_{L}(\varepsilon)-f_{R}(\varepsilon)$ due to higher temperature would also overlap with the effective transmission coefficient of antibonding state channel in configuration 1 , which makes the measured current $I$ different from $I_{-}$[see $I_{-}$and $I$ for $\epsilon_{-}=65,120 \Gamma$ in Fig. 2(b)]. In Fig. 2(c), we consider stronger couplings to the leads, $t_{c}=-6 \Gamma, \mu_{L}=12.5 \Gamma$ and $\mu_{R}=-12.5 \Gamma$. It shows that the stronger couplings to the leads make the level broadenings larger and the transmission coefficients wider. Then, the transmission coefficient of the bonding state channel results in different $I_{-}$under different configurations. In addition, the wider transmission coefficient of the antibonding state channel enhances the contribution of the antibonding current in configuration 1 and thus makes $I$ different from $I_{-}$[see $I_{-}$and $I$ for $\epsilon_{-}=6.5,12 \Gamma$ in Fig. 2(c)]. As a result, for the higher temperature or the stronger coupling to leads, the analysis given in Ref. [27] may become not applicable.

\section{REDUCED DENSITY MATRIX ELEMENTS AND THE CORRESPONDING TRANSPORT CURRENTS}

The experiment in Ref. [27] was performed under wide band limit, weak coupling, and large bias regime, which is a typical regime for transport experiment of DQDs devices. As shown in Eq. (8), the steady-state single-particle reduced density matrix in the wide-band limit is simply given by $\rho^{(1)}(t \rightarrow \infty)=\boldsymbol{v}(t, t \rightarrow \infty)$. Because of the indirect interdot weak coupling (small $a_{L, R}$ ), we can ignore the higher-order terms of $a_{L, R}$. The steady-state diagonal elements $v_{ \pm \pm}$then have the simple forms as

$$
\begin{aligned}
v_{ \pm \pm} & \simeq \int_{-\infty}^{+\infty} \frac{d \varepsilon}{2 \pi} \sum_{\alpha=L, R} f_{\alpha}(\varepsilon) \Gamma_{\alpha \pm \pm}\left|G_{ \pm \pm}^{r}(\varepsilon)\right|^{2} \\
& \simeq v_{L \pm \pm}+v_{R \pm \pm} .
\end{aligned}
$$

The steady-state transport currents through the bonding and antibonding state channels given in Eq. (25a) can be approximately expressed in terms of $v_{\alpha \pm \pm}$ :

$$
I_{ \pm} \simeq e \Gamma_{R \pm \pm} v_{L \pm \pm}-e \Gamma_{L \pm \pm} v_{R \pm \pm} .
$$

From the above results, we obtain the relations between occupation numbers of the bonding and antibonding states and the corresponding transport currents approximately:

$$
\rho_{ \pm \pm}^{(1)} \simeq \frac{I_{ \pm}}{e \Gamma_{R \pm \pm}} .
$$

We compare this approximated solution with the exact one given by Eqs. (15) and (16) at the steady-state limit $t \rightarrow \infty$. The results are presented in Fig. 3(a), where the energy configuration $\epsilon_{-}=-40 \Gamma$ is chosen as an example. As one can see, the approximation solution is almost the same as the (a)

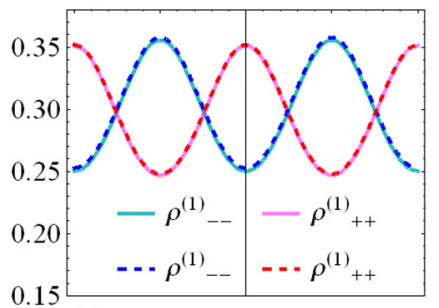

(c)

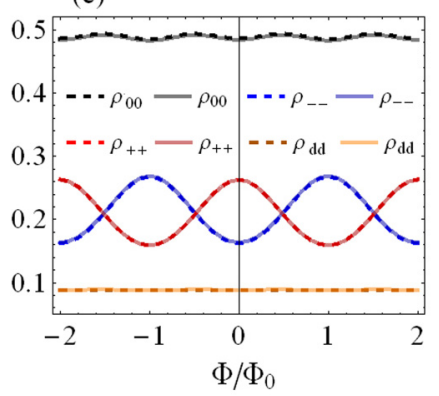

(b)

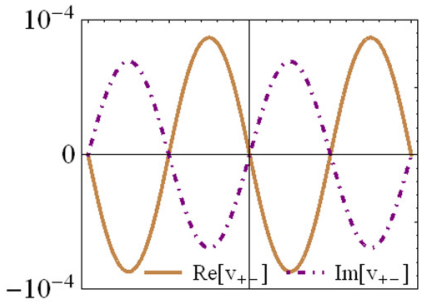

(d)

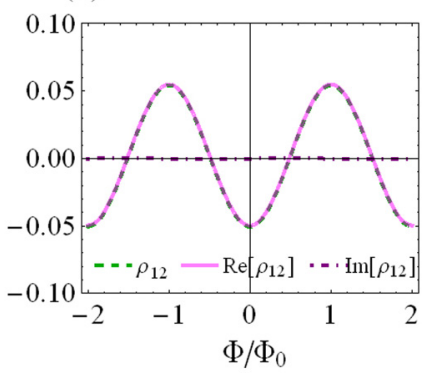

FIG. 3. (a) The exact (dashed lines) and approximate (solid lines) occupation numbers in the bonding and antibonding states given by Eqs. (8) and (28), respectively. (b) The real part and the imaginary part of $v_{+-}\left(\rho_{+-}\right)$. (c) The exact (dashed lines) and approximate (solid lines) diagonal elements of the reduced density matrix in the molecular basis, given by Eqs. (7) and (29), respectively. (d) The corresponding exact and approximate off-diagonal reduced density matrix elements in the dot basis.

exact one. Equation (28) implies that the currents through the bonding or antibonding state channels can be used to determine the particle occupations in the corresponding state. The bonding and antibonding state components of the Green function $\left|G_{ \pm \pm}^{r}(\varepsilon)\right|^{2}$ in Eq. (26) have sharp peaks located at $\epsilon_{ \pm}$, respectively, as shown in Fig. 2. When the bias is large $\left(\epsilon_{ \pm} \gg\right.$ $\left.\mu_{R}\right), v_{R \pm \pm}$ are ignorable. This is because electrons in the right lead hardly tunnel back into DQDs. The off-diagonal elements $v_{ \pm \mp}$ relate to the tunneling probability between the bonding and antibonding states. Because there is no direct coupling between the bonding and antibonding states, the electrons must hop to the leads, then hop back to the other state. The weak indirect interdot couplings suppress such probability, and hence $v_{ \pm \mp}$ are ignorable, as shown in Fig. 3(b) in which the magnitude of $v_{+-}$is the order of $10^{-3}$ of the magnitude of the diagonal elements. Consequently, the reduced density matrix of Eq. (17) in the steady-state limit can be approximately given by the bonding and antibonding currents:

$$
\begin{aligned}
\rho_{00} & \simeq\left(1-\frac{I_{+}}{e \Gamma_{R++}}\right)\left(1-\frac{I_{-}}{e \Gamma_{R--}}\right), \\
\rho_{--} & \simeq \frac{I_{-}}{e \Gamma_{R--}}\left(1-\frac{I_{+}}{e \Gamma_{R++}}\right), \\
\rho_{++} & \simeq \frac{I_{+}}{e \Gamma_{R++}}\left(1-\frac{I_{-}}{e \Gamma_{R--}}\right), \\
\rho_{d d} & \simeq \frac{I_{+}}{e \Gamma_{R++}} \frac{I_{-}}{e \Gamma_{R--}}, \\
\rho_{+-} & =v_{+-} \simeq 0 .
\end{aligned}
$$


We compare the above approximated solution with the exact elements of Eq. (17) in the steady-state limit in Fig. 3(c), which gives almost the same results between the approximated solution and the exact one.

For the practical application of DQDs as a promising qubit, one is interested in the quantum coherence between the two physical dots, which is described by the off-diagonal matrix element $\rho_{12}(t)$ in the physical dot basis. The reduced density matrix elements in the physical dot basis (the charge qubit basis) of the DQDs is given by the following relations $[19,28]$ :

$$
\begin{aligned}
\rho_{12}(t) & =\frac{1}{2}\left[\rho_{--}(t)-\rho_{++}(t)\right]+i \operatorname{Im} \rho_{+-}(t) \\
& \simeq \frac{1}{2}\left[\rho_{--}(t)-\rho_{++}(t)\right], \\
\rho_{22}^{11}(t) & =\frac{1}{2}\left[\rho_{--}(t)+\rho_{++}(t)\right] \pm \operatorname{Re} \rho_{+-}(t) \\
& \simeq \frac{1}{2}\left[\rho_{--}(t)+\rho_{++}(t)\right] .
\end{aligned}
$$

The off-diagonal element $\rho_{12}(t)$ is presented in Fig. 3(d). In the charge qubit basis, the probability of the diagonal elements $\rho_{11}(t)$ and $\rho_{22}(t)$ can also be determined from the diagonal density matrix elements $\rho_{--}$and $\rho_{++}$of the bonding and antibonding states, as shown in the above equation. Note that practically the currents flowing through the bonding and antibonding states cannot be simultaneously measured. The bonding state current $I_{-}$can be measured directly through the configuration 1 of Fig. 1(a). The antibonding state current $I_{+}$ is determined from the total current measured in configuration 2 of Fig. 1(a) when the conditions are satisfied such that $I_{-}$are almost the same in the two configurations (see the detailed analysis in Sec. III). Thus, the complete information of the reduced density matrix of the DQDs can be obtained experimentally from the currents through the relations given by Eqs. (29) and (30).

Next, we investigate the $A B$ oscillations in the reduced density matrix and the measured currents under different device geometry. The device geometry determines not only the parity of the bonding and antibonding state channels, but also the corresponding wave functions. The different device geometries are controlled by different signs of the direct interdot coupling $t_{c}$ and the indirect coupling parameters $a_{L}, a_{R}$. Different sings of $t_{c}$ correspond to different parities, and hence the $\mathrm{AB}$ phase dependencies of the bonding and antibonding states are changed by a half of period difference. When one changes the sign of $a_{L}$ or $a_{R}$, the $\mathrm{AB}$ oscillation phases in the transport currents remain unchanged but the amplitudes are modified (see Ref. [27]). However, for the reduced density matrix elements of the bonding and antibonding states, we find that not only their AB oscillation amplitudes are modified, but also the $A B$ phase dependence of each state is changed by a half of period difference. In Fig. 4, the AB phase dependencies of the transport currents and the diagonal density matrix elements are plotted for $a_{L} a_{R}<0$ and $a_{L} a_{R}>0$.

When the signs of $a_{L}$ and $a_{R}$ are the same, the level broadenings of the left or right lead coupled to the bonding or antibonding states, which are given by $\Gamma_{\alpha \pm \pm}=\Gamma_{\alpha}(1 \mp$ $\left.a_{\alpha} \cos \frac{\varphi}{2}\right), \alpha=L$ or $R$, enhance or shrink simultaneously for different magnetic flux. The AB oscillation amplitude of the transport current, which relies on $\Gamma_{L \pm \pm} \Gamma_{R \pm \pm}$ [see Eq. (25a)] is thus sensitive to the flux. The $\mathrm{AB}$ oscillation amplitudes of
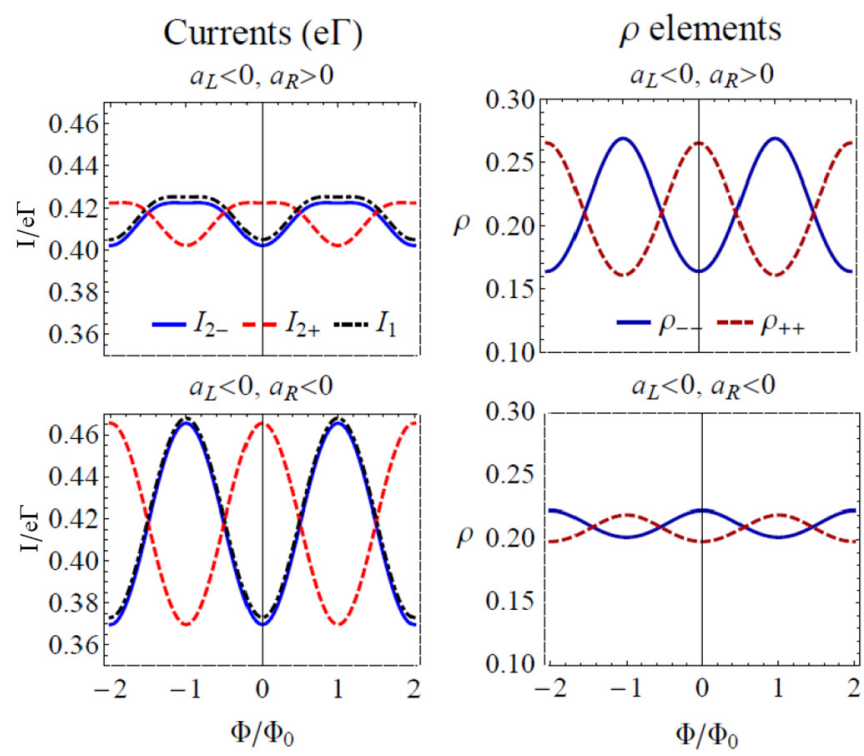

FIG. 4. The $\mathrm{AB}$ phase dependencies of $I_{2-}, I_{2+}$, and $I_{1}$ are plotted in the left column in unit $e \Gamma$ for different signs of $\alpha_{L}$ and $\alpha_{R}$. The AB phase dependencies of $\rho_{--}$and $\rho_{++}$are plotted in the right column. The other parameters are given according to Ref. [27].

currents for $a_{L} a_{R}>0$ are larger than those for $a_{L} a_{R}<0$ (see the left column in Fig. 4). This is because when the signs of $a_{L}$ and $a_{R}$ are different, the level broadenings for the bonding or antibonding states are enhanced only for one of the two leads at the same time. The corresponding bonding or antibonding current becomes less sensitive to the flux, as shown in Fig. 4. Meanwhile, because the different signs of $a_{L}$ and $a_{R}$ enhance the level broadening only for one of two leads forces electrons to localize at the bonding or antibonding states so that the $\mathrm{AB}$ oscillation amplitudes of $\rho_{--}$and $\rho_{++}$change opposite to that of the corresponding currents. Thus, the smaller amplitudes of the $\mathrm{AB}$ oscillation in the transport currents must correspond to the larger amplitudes in $\rho_{++}$and $\rho_{--}$, and vice versa (see Fig. 4).

Also, note that the AB oscillation phases of $\rho_{--}\left(\rho_{++}\right)$ for $a_{R}<0$ have a half-period difference from the case with $a_{R}>0$, as shown in the right column of Fig. 4. We compare the results at the magnetic flux $\Phi=0.5 \Phi_{0}$ and $0 \Phi_{0}$. When $\Phi=0.5 \Phi_{0}, \Gamma_{L \pm \pm}=\Gamma_{R \pm \pm}$. The symmetric energy configuration causes electrons to occupy both states with similar probabilities. When $\Phi=0 \Phi_{0}, \Gamma_{R--}$ is stronger for $a_{R}>0$ and weaker for $a_{R}<0$ than $\Gamma_{R--}$ at $\Phi=0.5 \Phi_{0}$. The stronger (weaker) $\Gamma_{R--}$ for $a_{R}>0\left(a_{R}<0\right)$ makes the electron in the bonding state hop to the right lead more easily (more difficultly), which gives rise to the smaller (larger) probability $\rho_{--}$of the bonding state than that at $\Phi=0.5 \Phi_{0}$. The AB phase dependence of $\Gamma_{L(R)++}$ is opposite to $\Gamma_{L(R)--}$, so that the AB oscillation dependence on $\rho_{++}$is exchanged with $\rho_{--}$when the sign of $a_{R}$ is changed, as shown in Fig. 4. This shows that the effect of changing the sign of $a_{R}$ (or $a_{L}$ ) is the same as changing the sign of $t_{c}$.

If the energy levels of the bonding and antibonding states are equally close to the center of the bias window, the traveling electrons passing through the corresponding channels have similar contribution to the reduced density matrix elements. 
(a) $a_{L}=-0.4, a_{R}=0.6$
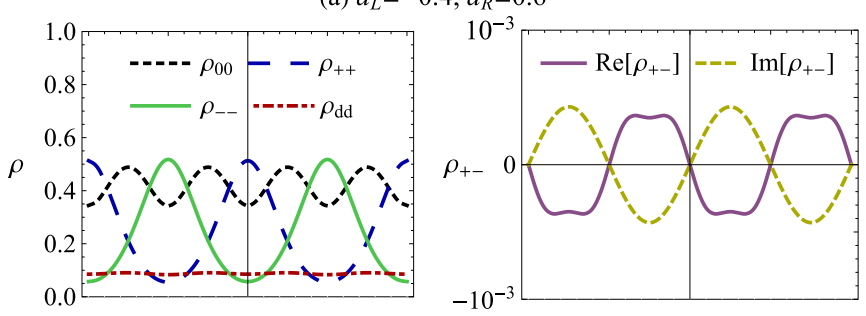

(b) $a_{L}=-0.6, a_{R}=0.9$
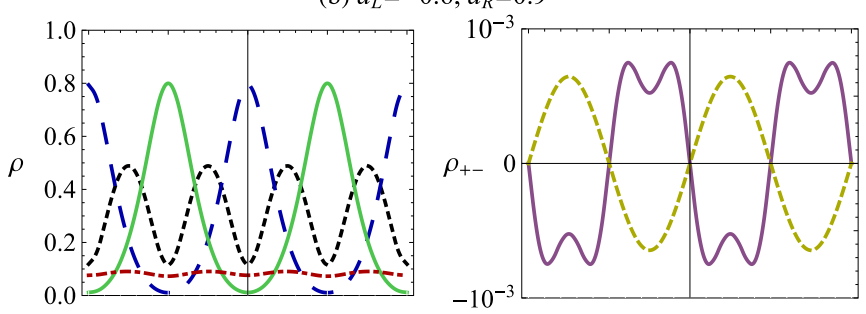

(c) $a_{L}=-1.0, a_{R}=1.0$
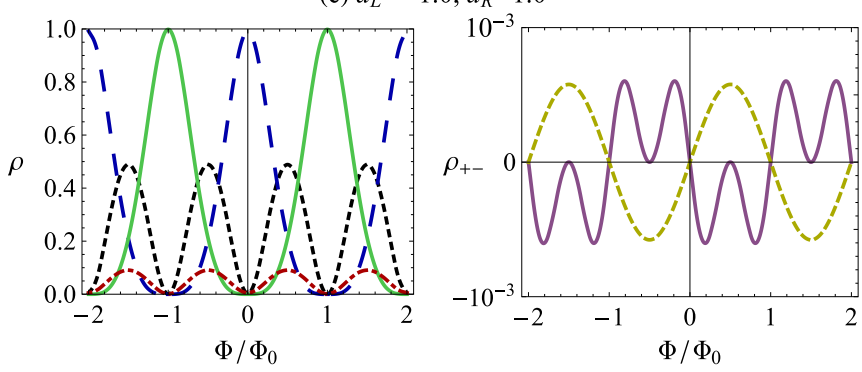

FIG. 5. The AB phase dependencies of all the reduced density matrix elements when $\epsilon_{-}=-60 \Gamma$. The different magnitudes of $a_{L, R}$ are taken here for the larger amplitudes of the $\mathrm{AB}$ phase dependencies. (a) $a_{L}=-0.4, a_{R}=0.6$, (b) $a_{L}=-0.6, a_{R}=0.9$, (c) $a_{L}=-1.0$, $a_{R}=1.0$. The other parameters are taken according to Ref. [27].

The larger $\alpha_{L, R}$ makes the electrons that flow from the left lead to the right lead more sensitive on the magnetic flux, and thus gives the larger amplitudes to the reduced density matrix elements $\rho_{++}$and $\rho_{--}$, as shown in Fig. 5. The DQDs system would have large probability to be in the bonding state or antibonding state at certain $\Phi$. For example, the DQDs system mainly locates in the antibonding state (bonding state) at $\Phi=0\left(\Phi / \Phi_{0}=1\right)$. Therefore, if a strong indirect coupling can be practically succeeded, it could provide an efficient way to manipulate the probabilities of the bonding and antibonding states through the magnetic flux. On the other hand, the right column of Fig. 5 presents the off-diagonal matrix element $\rho_{+-}$as a function of the AB flux. The result shows that even for strong indirect coupling $a_{L, R}$, the amplitude of $\rho_{+-}$ is still very small (the order of $10^{-3}$ in comparison with the diagonal element $\rho_{++}$and $\rho_{--}$). This small off-diagonal matrix element is due to the large direct interdot coupling $t_{c}$ taken in the experiment [27] (reducing the direct interdot coupling, one should see the strong interference effect between the bonding and antibonding states as the Fano resonance effect in the strong indirect coupling regime $[25,26])$. Therefore, the approximate relations given by Eq. (30) are still valid, which can be used to determine all the reduced density matrix elements in the charge qubit basis. These analyses provide the full information for the reconstruction of quantum states through the measurement of transport currents.

\section{SUMMARY}

In conclusion, using the quantum transport theory based on the master equation [28-30], we have verified the validity of the method used in Ref. [27] for analyzing the transport currents passing through the bonding and antibonding state channels. We show that when the energy level of the bonding state is within a quite large range near the middle of the bias window, the bonding current in configuration 1 well approximates the bonding current in configuration 2 . However, in high temperature and/or the strong couplings between the dots and the leads, this analysis may not be valid. This is because the bias window is broadened in high temperature and the effective transmissions of the channels become wider for the strong couplings to the leads. We then explore the relation between the $\mathrm{AB}$ phase dependence of electron probabilities in the bonding and antibonding states and the corresponding currents. We find that the electron probabilities in the bonding and antibonding states can be directly obtained from the measured currents [see Eq. (29)], and the electron probabilities of the bonding and antibonding states can be used to determine the probabilities of the dot states (diagonal matrix element) and the coherence (the off-diagonal matrix element) between two dot states for charge qubit manipulations [see Eq. (30)]. We also find the $A B$ oscillation relation between the reduced density matrix elements and the transport currents of the bonding and antibonding states under different device geometries. These results provide not only the full information for the reconstruction of quantum dot states through the measurement of transport current, but also a practice way to manipulate quantum coherence between the two physical dot states with the $\mathrm{AB}$ magnetic flux.

\section{ACKNOWLEDGMENTS}

We thank A. Aharony and O. Entin-Wohlman for the fruitful discussions. This work is supported by the Ministry of Science and Technology, Taiwan, ROC, under Contract No. NSC1022112-M-006-016-MY3, by the Headquarters of University Advancement at the National Cheng Kung University, which is sponsored by the Ministry of Education, Taiwan, ROC. We acknowledge the computing resources of High Performance Computing Facility in the National Cheng Kung University.

\section{APPENDIX: EXACT MASTER EQUATION}

The system described in Sec. II can be treated as an open quantum device. The dynamics of the double dot is described by the reduced density matrix $\rho(t)$, which is obtained by tracing over all the degrees of freedom of the reservoirs from the total density matrix $\rho_{\text {tot }}(t)$ of the DQDs plus the leads: $\rho(t)=\operatorname{Tr}_{R}\left[\rho_{\text {tot }}(t)\right]$. The exact master equation which governs the dynamics of the DQDs has been derived [28,29,31]:

$$
\begin{aligned}
\frac{d \rho(t)}{d t}= & \frac{1}{i}\left[H_{S}^{\prime}(t), \rho(t)\right]+\sum_{i, j}\left\{\gamma _ { i j } ( t ) \left[2 d_{j} \rho(t) d_{i}^{\dagger}\right.\right. \\
& \left.-d_{i}^{\dagger} d_{j} \rho(t)-\rho(t) d_{i}^{\dagger} d_{j}\right]+\widetilde{\gamma}_{i j}(t)\left[d_{i}^{\dagger} \rho(t) d_{j}\right. \\
& \left.\left.-d_{j} \rho(t) d_{i}^{\dagger}+d_{i}^{\dagger} d_{j} \rho(t)-\rho(t) d_{j} d_{i}^{\dagger}\right]\right\},
\end{aligned}
$$


where $H_{S}^{\prime}(t)=\sum_{i, j} \epsilon_{i j}^{\prime}(t) d_{i}^{\dagger} d_{j}$ is an effective Hamiltonian and $\epsilon_{i j}^{\prime}(t)$ is the renormalized time-dependent energy level $(i=j)$ or the shifted interdot transition amplitude $(i \neq j)$ between the DQDs. All the time-dependent coefficients in Eq. (A1) are determined by the retarded Green function $\boldsymbol{u}\left(t, t_{0}\right)$ and the correlation Green function $\boldsymbol{v}(\tau, t)$ in Keldysh's nonequilibrium Green function technique [29]. Explicitly, the renormalized energy levels of DQDs $\boldsymbol{\epsilon}^{\prime}(t)$, the dissipation coefficient $\boldsymbol{\gamma}(t)$, and the fluctuation coefficient $\widetilde{\gamma}(t)$ are given by

$$
\begin{aligned}
\boldsymbol{\epsilon}^{\prime}(t) & =\frac{1}{2}\left[\dot{\boldsymbol{u}}(t) \boldsymbol{u}^{-1}(t)-\text { H.c }\right], \\
\boldsymbol{\gamma}(t) & =-\frac{1}{2}\left[\dot{\boldsymbol{u}}(t) \boldsymbol{u}^{-1}(t)+\text { H.c }\right], \\
\tilde{\boldsymbol{\gamma}}(t) & =\dot{\boldsymbol{v}}(t)-\left[\dot{\boldsymbol{u}}(t) \boldsymbol{u}^{-1}(t) \boldsymbol{v}(t)+\text { H.c }\right],
\end{aligned}
$$

where $\boldsymbol{u}(t) \equiv \boldsymbol{u}\left(t, t_{0}\right)$ and $\boldsymbol{v}(t) \equiv \boldsymbol{v}(t, t)$ are the nonequilibrium Green functions. The Green function $\boldsymbol{u}\left(t, t_{0}\right)$ obeys the following integrodifferential equation:

$$
\frac{\partial}{\partial t} \boldsymbol{u}\left(t, t_{0}\right)+i \boldsymbol{\epsilon} \boldsymbol{u}\left(t, t_{0}\right)+\sum_{\alpha} \int_{t_{0}}^{t} d \tau \boldsymbol{g}_{\alpha}(t, \tau) \boldsymbol{u}\left(\tau, t_{0}\right)=0
$$

and $\boldsymbol{v}(\tau, t)$ is given by [29-31]

$$
\boldsymbol{v}(\tau, t)=\int_{t_{0}}^{\tau} d \tau_{1} \int_{t_{0}}^{t} d \tau_{2} \sum_{\alpha} \boldsymbol{u}\left(\tau, \tau_{1}\right) \widetilde{\boldsymbol{g}}_{\alpha}\left(\tau_{1}, \tau_{2}\right) \boldsymbol{u}^{\dagger}\left(t, \tau_{2}\right) .
$$

The integral kernels in the above equations are

$$
\begin{aligned}
& \boldsymbol{g}_{\alpha}(t, \tau)=\int_{-\infty}^{\infty} \frac{d \varepsilon}{2 \pi} \boldsymbol{\Gamma}_{\alpha}(\varepsilon) e^{-i \varepsilon(t-\tau)}, \\
& \widetilde{\boldsymbol{g}}_{\alpha}(t, \tau)=\int_{-\infty}^{\infty} \frac{d \varepsilon}{2 \pi} \boldsymbol{\Gamma}_{\alpha}(\varepsilon) f_{\alpha}(\varepsilon) e^{-i \varepsilon(t-\tau)},
\end{aligned}
$$

with

$$
\Gamma_{\alpha i j}(\varepsilon)=2 \pi \sum_{k} V_{i \alpha k} V_{j \alpha k}^{*} \delta\left(\varepsilon-\varepsilon_{\alpha k}\right) .
$$

Here, $\boldsymbol{\Gamma}_{\alpha}(\varepsilon)$ is called the spectral density (level broadening) of lead $\alpha$, and $f_{\alpha}(\varepsilon)=1 /\left[e^{\beta\left(\varepsilon-\mu_{\alpha}\right)}+1\right]$ is the corresponding Fermi-Dirac distribution function with the chemical potential $\mu_{\alpha}$ and the initial reservoir temperature $\beta=1 / k_{B} T$. The exact solution of the above master equation for the DQDs is given by Eq. (7).
[1] D. Loss and D. P. DiVincenzo, Phys. Rev. A 57, 120 (1998).

[2] T. Hayashi, T. Fujisawa, H. D. Cheong, Y. H. Jeong, and Y. Hirayama, Phys. Rev. Lett. 91, 226804 (2003).

[3] J. M. Elzerman, R. Hanson, J. S. Greidanus, L. H. Willems van Beveren, S. De Franceschi, L. M. K. Vandersypen, S. Tarucha, and L. P. Kouwenhoven, Phys. Rev. B 67, 161308(R) (2003).

[4] J. R. Petta, A. C. Johnson, C. M. Marcus, M. P. Hanson, and A. C. Gossard, Phys. Rev. Lett. 93, 186802 (2004).

[5] J. Gorman, D. G. Hasko, and D. A. Williams, Phys. Rev. Lett. 95, 090502 (2005).

[6] A. C. Johnson, J. R. Petta, J. M. Taylor, A. Yacoby, M. D. Lukin, C. M. Marcus, M. P. Hanson, and A. C. Gossard, Nature (London) 435, 925 (2005).

[7] J. R. Petta, A. C. Johnson, J. M. Taylor, E. A. Laird, A. Yacoby, M. D. Lukin, C. M. Marcus, M. P. Hanson, and A. C. Gossard, Science 309, 2180 (2005).

[8] S. Foletti, H. Bluhm, D. Mahalu, V. Umansky, and A. Yacoby, Nat. Phys. 5, 903 (2009).

[9] K. D. Petersson, C. G. Smith, D. Anderson, P. Atkinson, G. A. C. Jones, and D. A. Ritchie, Nano Lett. 10, 2789 (2010).

[10] B. M. Maune, M. G. Borselli, B. Huang, T. D. Ladd, P. W. Deelman, K. S. Holabird, A. A. Kiselev, I. Alvarado-Rodriguez, R. S. Ross, A. E. Schmitz, M. Sokolich, C. A. Watson, M. F. Gyure, and A. T. Hunter, Nature (London) 481, 344 (2012).

[11] L. Fricke, M. Wulf, B. Kaestner, V. Kashcheyevs, J. Timoshenko, P. Nazarov, F. Hohls, P. Mirovsky, B. Mackrodt, R. Dolata, T. Weimann, K. Pierz, and H. W. Schumacher, Phys. Rev. Lett. 110, 126803 (2013).

[12] Z. Shi, C. B. Simmons, D. R. Ward, J. R. Prance, X. Wu, T. S. Koh, J. K. Gamble, D. E. Savage, M. G. Lagally, M. Friesen, S. N. Coppersmith, and M. A. Eriksson, Nat. Commun. 5, 3020 (2014).
[13] T. Fujisawa, T. Hayashi, and S. Sasaki, Rep. Prog. Phys. 69, 759 (2006).

[14] R. Hanson, J. R. Petta, S. Tarucha, and L. M. K. Vandersypen, Rev. Mod. Phys. 79, 1217 (2007).

[15] P. Samuelsson and M. Büttiker, Phys. Rev. B 73, 041305(R) (2006).

[16] J. Medford, J. Beil, J. M. Taylor, S. D. Bartlett, A. C. Doherty, E. I. Rashba, D. P. DiVincenzo, H. Lu, A. C. Gossard, and C. M. Marcus, Nat. Nanotechnol. 8, 654 (2013).

[17] D. Kim, Z. Shi, C. B. Simmons, D. R. Ward, J. R. Prance, T. S Koh, J. K. Gamble, D. E. Savage, M. G. Lagally, M. Friesen, S. N. Coppersmith, and M. A. Eriksson, Nature (London) 511, 70 (2014).

[18] M. W. Y. Tu, W. M. Zhang, and J. Jin, Phys. Rev. B 83, 115318 (2011).

[19] M. W. Y. Tu, W. M. Zhang, and F. Nori, Phys. Rev. B 86, 195403 (2012).

[20] Y. Aharonov and D. Bohm, Phys. Rev. 115, 485 (1959).

[21] A. W. Holleitner, C. R. Decker, H. Qin, K. Eberl, and R. H. Blick, Phys. Rev. Lett. 87, 256802 (2001).

[22] T. Hatano, M. Stopa, W. Izumida, T. Yamaguchi, T. Ota, and S. Tarucha, Phys. E (Amsterdam) 22, 534 (2004).

[23] M. Sigrist, A. Fuhrer, T. Ihn, K. Ensslin, S. E. Ulloa, W. Wegscheider, and M. Bichler, Phys. Rev. Lett. 93, 066802 (2004).

[24] D. Loss and E. V. Sukhorukov, Phys. Rev. Lett. 84, 1035 (2000).

[25] K. Kang and S. Y. Cho, J. Phys.: Condens. Matter 16, 117 (2004).

[26] T. Kubo, Y. Tokura, T. Hatano, and S. Tarucha, Phys. Rev. B 74, 205310 (2006).

[27] T. Hatano, T. Kubo, Y. Tokura, S. Amaha, S. Teraoka, and S. Tarucha, Phys. Rev. Lett. 106, 076801 (2011).

[28] M. W. Y. Tu and W. M. Zhang, Phys. Rev. B 78, 235311 (2008). 
[29] J. S. Jin, M. W. Y. Tu, W. M. Zhang, and Y. J. Yan, New J. Phys. 12, 083013 (2010).

[30] M. W. Y. Tu, W. M. Zhang, J. Jin, O. Entin-Wohlman, and A. Aharony, Phys. Rev. B 86, 115453 (2012).

[31] P. Y. Yang, C. Y. Lin, and W. M. Zhang, Phys. Rev. B 92, 165403 (2015).
[32] We thank C. U Lei and also C. Y. Lin for providing this unpublished solution; also see the derivation given later by M. W. Y. Tu, J. H. Liu, and W. M. Zhang, arXiv:1503.05376.

[33] H. Haug and A. P. Jauho, in Quantum Kinetics in Transport and Optics of Semiconductors, Springer Series in Solid-State Sciences, 2nd ed. (Springer, Berlin, 2008), Vol. 123. 